\title{
A Dilemma for Viruses and Giant Viruses: Which Endocytic Pathway to Use to Enter Cells?
}

\author{
Eric Ghigo \\ URMITE CNRS UMR 6236-IRD, Université de la Méditerranée, Marseille, France
}

\section{Key Words}

Acanthamoeba polyphaga mimivirus - Endocytosis •

Phagocytosis • Viral entry

\begin{abstract}
Viruses must enter host cells to deliver their genetic material and accessory proteins. Endocytosis offers to viruses the opportunity to enter host cells. However, endocytosis is a complex phenomenon that includes different mechanisms, clathrin-mediated endocytosis, caveolin-mediated endocytosis, macropinocytosis, and phagocytosis. Here, I describe the ways used by different viruses to exploit these endocytic pathways.

Copyright $\odot 2010$ S. Karger AG, Basel
\end{abstract}

\section{Introduction}

As obligate intracellular pathogens, all mammalian viruses must have ways of entering target cells to initiate replication and infection. Viruses have therefore evolved a variety of mechanisms to deliver their genes and accessory proteins into host cells. The first step of viral invasion consists of passing through the host cell's plasma membrane, which is a major barrier for invading agents. Viruses can enter a cell in two ways: by direct fusion with the cell plasma membrane (not reviewed here) or by internalization through endocytosis. Endocytosis offers many benefits to viruses. These benefits include fast transit through plasma membranes and cells, the low $\mathrm{pH}$ of the endosomes that is required for some viruses with a conformational dependence change, and the possibility of being delivered near the nuclear pore for nuclear-replicating viruses. Consequently, a majority of viruses use endocytosis to enter cells. Several endocytic pathways have been described, differing in the size of the endocytic vesicles, the nature of the cargo, and the mechanism of vesicle formation. Endocytosis pathways include mechanisms involved in the internalization of small volumes, such as clathrin- or caveolin-mediated endocytosis, and mechanisms involved in the internalization of large volumes, such as macropinocytosis or phagocytosis (Box 1). In this review, I will describe the general mechanism process of each endocytic pathway in eukaryotic cells and give examples of the entry styles of mammalian viruses (fig. 1).

\section{Clathrin-Mediated Endocytosis}

Of the endocytic pathways used by viruses, the clathrin endocytic route is the most common [1]. Clathrin-mediated endocytosis (CME) is a rapid and efficient process that occurs constitutively and continuously in all mam-

\section{KARGER}

Fax +4161306 1234

E-Mail karger@karger.ch

www.karger.com
(C) 2010 S. Karger AG, Basel

$0300-5526 / 10 / 0535-0274 \$ 26.00 / 0$

Accessible online at:

www.karger.com/int
Eric Ghigo

URMITE CNRS UMR 6236-IRD 3R198

Faculté de Médecine, $27 \mathrm{Bd}$. Jean Moulin

FR-13385 Marseille Cedex 05 (France)

Tel. +3349132 4375, Fax +3349183 0390, E-Mail eric.ghigo@ univmed.fr 
Box 1. Endocytic pathways

\section{Clathrin-mediated endocytosis}

CME is a rapid and efficient process that occurs constitutively and continuously in all mammalian cells. It is characterized by the clustering of ligated transmembrane receptors into clathrin-coated vesicles and is involved in the uptake of nutrients essential for cells, such as iron-laden transferrin, cholesterol-laden low-density lipoproteins, growth factors, antigens, and recycling receptors.

\section{Caveolin-mediated endocytosis}

The mechanism of clathrin-independent endocytosis (or caveolin-mediated endocytosis) acts via caveolae to internalize extracellular ligands (folic acid, albumin, autocrine motility factor) and bacterial toxins (cholera toxin, tetanus toxin), recycle membrane components (glycosphingolipids and GPI-anchored protein) and transcytose numerous transduction signals (such as calcium signaling).

\section{Macropinocytosis}

Macropinocytosis is a process used by cells to trap large amounts of macromolecules and fluids, and occurs in many cells upon stimulation by mediators such as growth factors, interleukin-4 and phorbol esters. Macropinocytotic functions are diverse; it is involved in cell migration, antigen presentation, immune surveillance, and may play a role in the control of activated signaling molecules.

\section{Phagocytosis}

Phagocytosis consists of the ingestion of particles larger than $0.5 \mu \mathrm{m}$ by specialized cells. In mammals, professional phagocytes such as macrophages, monocytes, dendritic cells and neutrophils possess the microbicidal machinery that allows them to eliminate a great majority of bacteria, fungi, and protozoa. Phagocytosis is a receptor-mediated endocytosis process.

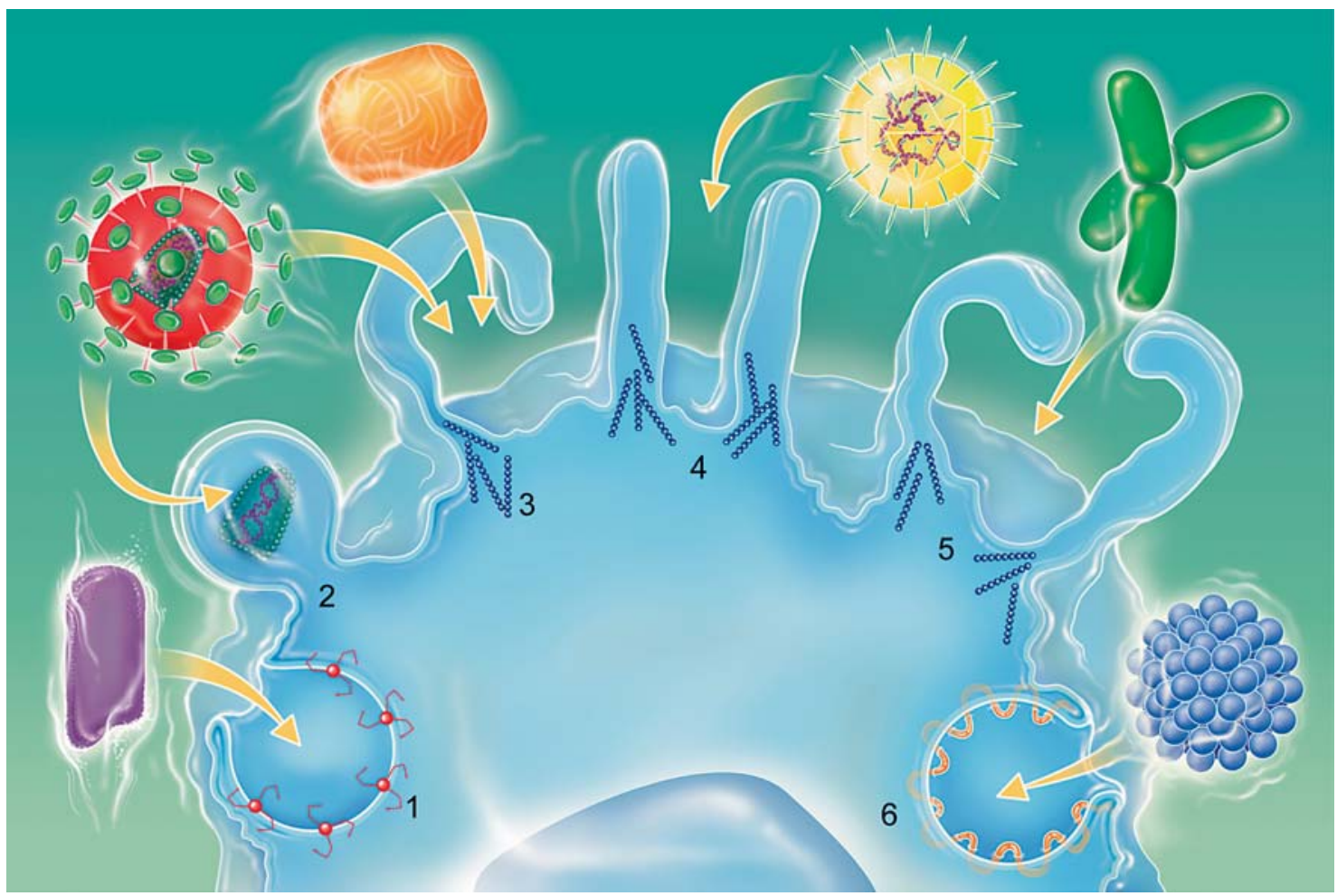

Fig. 1. Multiple portals of virus entry into mammalian cells. (1) Clathrin-mediated entry (e.g. vesicular stomatitis virus). (2) Fusion-entry (e.g. HIV). (3) Macropinocytosis-mediated entry (e.g. vaccinia virus). (4) Phagocytosis-like-mediated entry (e.g. herpes simplex virus). (5) Phagocytosis-mediated entry (e.g. bacteria). (6) Caveolin-mediated entry (e.g. simian virus 40). The virus entry by late endosome-mediated endocytosis is not illustrated here. Illustration under Creative Commons Attribution License from [56]. 
malian cells. CME is well known and is characterized by the clustering of ligated transmembrane receptors into clathrin-coated vesicles (CCV) of about $120 \mathrm{~nm}$. CME is involved in the uptake of nutrients essential for cells, such as iron-laden transferrin, cholesterol-laden low-density lipoproteins, growth factors, antigens, and recycling receptors [2]. CCV formation occurs in four steps and requires several molecules, such as small G-proteins (Rab5, Arf6, etc.), adaptor proteins (AP2, AP180, etc.), accessory proteins (Eps15, amphiphysin, dynamin, endophilin, actin, etc.), and phosphatidylinositols. Genetic studies in multicellular model organisms have suggested that some of these proteins may not be essential for CME in all cells [3]. The first step is the assembly of a clathrin-coated pit and bud formation on the cytoplasmic face of the plasma membrane by recruiting adaptor and accessory proteins, such as amphiphysin. The second step is clathrin-coated pit maturation, which involves actin, dynamin, and endophilin and leads to a fully formed and constricted pit that is still associated with the cytoplasmic face of the plasma membrane. The third step is fission, which requires dynamin, an atypically large and modular GTPase [3]. The final stage is CCV uncoating [2-4] and the delivery of the CCV contents to early endosomes. Inhibition of $\mathrm{CME}$ is possible using dominant-negative expression constructs, including Eps15 [5] and K44A-dynamin [6], and treatment with drugs such as chloropromazine [7].

Several viruses (table 1), including vesicular stomatitis virus, influenza A virus, Semliki Forest virus, adenovirus 2 (Ad2), and adenovirus 5 (Ad5), enter cells via CME [8]. Here, we focus on the entry of two viruses, adenovirus (Ad2 and Ad5) and influenza A.

Ad2/Ad5 are non-enveloped DNA viruses with an icosahedral capsid decorated with fiber proteins that infect the respiratory epithelium. The capsid fiber proteins bind to coxsackie adenovirus receptor, a host cell glycoprotein [9]. The signaling event resulting from clustering of the integrins due to coxsackie adenovirus receptor ligation activates PI3 kinases, Rho GTPase (Cdc42, Rac), docking protein $130^{\text {cas }}$, Rab5 recruitment, and cytoskeleton rearrangements; Ad2/Ad5 is subsequently internalized by a CCV [9-11]. Once in a CCV, viruses are delivered to the slightly acidic ( $\mathrm{pH}$ 6.0) early endosome. At pH 6.0, Ad2/ Ad5 undergo a conformational change, allowing viruses to escape from the early endosomes and reach the host cell cytosol. Then, viruses reach the nuclear pores using the microtubule network as a motorway. The dynein/ dynactin motor complex is required for virus transport into the cytosol and to the nuclear pore, where viruses deliver their genetic material [12].
Influenza virus A is an enveloped negative-strained RNA virus. Its hemagglutinin protein interacts with specific sialic acids during attachment and entry into host cells [13]. Once internalized through clathrin-coated pits, viral particles are trafficked along the endocytic pathway from early endosomes to the acidified late endosomes (pH 5.0). Several Rab proteins, such as Rab5 and Rab7, are involved in influenza virus trafficking because dominant-negative mutants of either Rab5 or Rab7 significantly inhibit viral infectivity, suggesting that both early and late endosomes are required [14]. The acidic environment of the late endosomes activates the M2 protein ion channel in the viral membrane, allowing the internal capsid to be acidified, and triggers a hemagglutinin protein-catalyzed fusion between the viral and endosomal membranes. Viruses are delivered to the host cell cytosol [10, 15]. Then, the genetic material is imported through importin $\beta$ and delivered into the nucleus for viral gene expression and replication. In addition, ubiquitination and vacuolar protein sorting machinery are necessary for endosomal sorting of the influenza virus [16].

\section{Caveolin-Mediated Endocytosis}

The mechanism of a clathrin-independent endocytosis, or so-called caveolin-mediated endocytosis, has begun to be elucidated. Caveolin-mediated endocytosis acts via caveolae to internalize extracellular ligands (folic acid, albumin, autocrine motility factor) and bacterial toxins (cholera toxin, tetanus toxin), recycle membrane components (glycosphingolipids and GPI-anchored protein), and transcytose numerous transduction signals (such as calcium signaling). Caveolae are flask-shaped invaginations, $50-80 \mathrm{~nm}$ in diameter, that are present at the plasma membrane of many cell types [17]. Caveolin is synthesized in the endoplasmic reticulum and then transported to the Golgi apparatus, where it is oligomerized and associates with cholesterol and glycosphingolipid-rich lipid-raft domains to form caveolae [10, 17]. Caveolae are exported from the Golgi to the plasma membrane [17]. One caveolar structure is formed with at least 144 caveolin molecules. Caveolae are composed of glycosphingolipids such as gangliosides GM1 and GM3, sphingomyelin, and more than 20,000 other molecules [18]. How caveolin assembles into a caveolar structure is still unclear; however, the molecules and mechanism involved in the exit of the caveolae from the Golgi apparatus have been elucidated. Syntaxin-6 (a SNARE protein) is involved in delivering caveolin, GPI-anchored protein, and 
Table 1. Entry pathways of some viruses

\begin{tabular}{lll}
\hline Viruses & Entry pathway & Ref. \\
\hline APMV & phagocytosis & {$[56]$} \\
\hline Adenovirus 2 & clathrin-mediated endocytosis & {$[8]$} \\
\hline Adenovirus 3 & macropinocytosis & {$[40-42]$} \\
\hline Adenovirus 5 & clathrin-mediated endocytosis & {$[8]$} \\
\hline Coxsackie B virus & caveolae-mediated endocytosis & {$[8,27]$} \\
\hline Ebolavirus & caveolae-mediated endocytosis & {$[72]$} \\
\hline Echo 1 virus & caveolae-mediated endocytosis & {$[8,27]$} \\
\hline HSV-1 & phagocytosis & {$[57]$} \\
\hline HIV-1 & plasma membrane/ & {$[43-45]$} \\
\hline macropinocytosis & \\
\hline Polyomavirus & clathrin-mediated endocytosis & {$[8]$} \\
\hline Semliki Forest virus & caveolae-mediated endocytosis & {$[73]$} \\
\hline Sindbis virus & clathrin-mediated endocytosis & {$[8]$} \\
\hline SV40 & caveolae-mediated endocytosis & {$[23]$} \\
\hline Vaccinia virus & macropinocytosis & {$[39]$} \\
\hline Vesicular stomatitis & $\begin{array}{l}\text { clathrin-mediated endocytosis/ } \\
\text { virus }\end{array}$ & {$[8,68]$} \\
& $\begin{array}{l}\text { degradative endosome-mediated } \\
\text { endocytotic process }\end{array}$ & \\
\hline
\end{tabular}

GM1 to the plasma membrane $[17,19]$. Caveolar endocytosis is stimulated by several ligands and leads to the formation of small caveolae vesicles that fuse with early endosomes or with each other to form caveosomes. These caveosomes can then fuse with the endoplasmic reticulum or Golgi apparatus. Dynamin, actin, and Src kinase are involved in the endocytosis of caveolae [17, 20, 21]. In contrast to early endosomes or CCVs, caveosomes are not decorated with EEA1 (early endosome autoantigen-1) and have a neutral $\mathrm{pH}[17,19,22,23]$.

Caveolae-mediated endocytosis is exploited by certain non-enveloped viruses (table 1 ), such as simian virus 40 (SV40), to enter cells [23]. SV40 bypasses the traditional endosome/lysosomes route because it is delivered directly from caveolae to the endoplasmic reticulum. Several steps of the SV40 caveolae-mediated entry have been elucidated. To enter cells, SV40 interacts with gangliosides and the major histocompatibility complex class I antigen [24, 25], which leads to its sequestration into caveolae [8]. Once the virus is in caveolae, a signal transduction cascade is initiated by the activation of tyrosine kinases. This activation induces local phosphorylation, depolymerization of F-actin, production of $\mathrm{Pi}(4,5) \mathrm{P}_{2}$, actin accumulation around the caveolae, dynamin 2 recruitment, and activation of ca- veolar endocytosis. These events are necessary for viruses to be internalized within caveolae $[8,19]$. Later on, SV40 viruses are delivered to the caveosomes. Caveosomes containing SV40 are transported to the endoplasmic reticulum via dynein-dependant microtubule mediated transport. The next step is the passage of viral particles from caveosomes to the endoplasmic reticulum $[22,23]$. This event is probably mediated by COPI (coat-omer complex I) and requires the GTPases Arf1 and Sarl $[23,26]$. Once in the endoplasmic reticulum, SV40 enters the host cell cytosol and reaches the nucleus. The endoplasmic reticulum-escaping mechanism is still unknown. Several other viruses use caveolin-mediated endocytosis, including the ECHO 1 virus and coxsackie B. Their entry into host cells seems to occur in caveosomes as found for SV40, but, in contrast to SV40, the endoplasmic reticulum is not involved in further steps of intracellular transport $[8,27]$.

\section{Macropinocytosis}

Macropinocytosis is a process used by cells to trap large amounts of macromolecules and fluids and occurs in many cells upon stimulation by mediators such as growth factors, interleukin-4, and phorbol esters [2830]. The mechanisms governing macropinocytosis are not very well known, and the strong likeness between macropinocytosis and phagocytosis makes a distinction difficult. Macropinocytotic functions are diverse; the process is likely involved in cell migration, antigen presentation, and immune surveillance, and it may play a role in the control of activated signaling molecules [3]. This endocytic pathway, which is independent of receptors and dynamin, is associated with actin-dependent plasma membrane ruffling $[8,28]$ and is inhibited by amiloride analogs [31,32]. Macropinocytosis leads to the formation of macropinosomes, which are large vesicles $(>1 \mu \mathrm{m})$ characterized by the presence of rabankyrin-5, a macropinosome marker [33]. The fate of macropinosomes depends on the cell type. Indeed, in macrophages, macropinosomes are converted into early endosomes, late endosomes, and then lysosomes where their content is degraded. In A431 cells, macropinosomes do not seem to interact with the classical endocytic pathway. In some cases, macropinosomes fuse again with the plasma membrane [28]. The mechanisms governing macropinocytosis are close to those that control the phagocytic process. Macropinocytotic mechanisms depend on actin remodeling, rho GTPase, Arf6, PI3K [34, 35] and Rac-dependent kinase PAK1 [36]. Rab 
proteins (Rab5, Rab34) are also involved in the formation of the macropinosomes $[37,38]$. Macropinocytosis can be specifically inhibited using amilorides such as ethyl-N-isopropyl amiloride (EIPA), a blocker of $\mathrm{Na}^{+} / \mathrm{H}^{+}$ exchange [31, 32].

In contrast to the clathrin- and caveolin-mediated entries, few viruses have been described as using macropinocytosis to infect target cells (table 1). They include the vaccinia virus, HIV1, and adenovirus 3 (Ad3). However, the exact mechanisms involved in macropinocytosis-mediated entry remain to be elucidated.

Several results suggest that the vaccinia virus, a large enveloped virus, uses macropinocytosis to enter cells [39]. The viral uptake is actin- and Rac1-dependent. The blockage of several molecules involved in macropinocytosis, such as Pak1, PI3K, Arf6, or $\mathrm{Na}^{+} / \mathrm{H}^{+}$ion exchanger, inhibits cell infection. In addition, the membrane of mature virions is enriched in phosphatidylserine, a phospholipid that is needed for the uptake of apoptotic debris by macropinocytosis. Mature virions seem to elicit the macropinocytic response in host cells by mimicking apoptotic bodies [39].

Ad3, a non-enveloped, double-stranded DNA virus, enters epithelial and hematopoietic cells by direct macropinocytosis, as demonstrated using a collection of inhibitors, siRNAs, and mutant proteins. Two processes are involved in the entry of Ad3 into host cells. Ad3 activates Rac1 and PI3K by binding to CD46 and by the clustering $\alpha \mathrm{v}$-integrin [40-42].

HIV1, an enveloped, single-stranded RNA lentivirus, is internalized by primary human macrophages and brain microvascular endothelial cells through macropinocytosis. Pak1 and its substrate, dynein light chain 1 , are required in the early stages of HIV-1 infection. HIV-1 entry is inhibited by amilorides. Electron microscopy shows that HIV resides within large-sized compartments with an irregular shape, likely macropinosomes. Although different authors suggest that HIV may enter and infect cells through macropinocytosis [43, 44], this mechanism of entry may be inefficient because most of the virus particles are degraded [45].

\section{Phagocytosis}

Phagocytosis consists of the ingestion of particles larger than $0.5 \mu \mathrm{m}$ by specialized cells [46]. In mammals, professional phagocytes such as macrophages, monocytes, dendritic cells and neutrophils possess the microbicidal machinery that allows them to eliminate a great majority of bacteria, fungi and protozoa. To discriminate among the multiple infectious agents they meet, myeloid cells possess a restricted number of phagocytic receptors.

The mechanisms underlying phagocytosis, a receptormediated endocytosis process, are complex, but some general principles can be discerned $[46,47]$. Phagocytosis is initiated by the interaction of cell surface receptors, called pattern recognition receptors, with their ligands (pathogens, cells, particles, etc.). Pattern recognition receptors include Fc receptors that recognize opsonized microorganisms; the complement receptor CR3 (CD11b/ $\mathrm{CD} 18)$ that recognizes opsonized and unopsonized organisms; the mannose receptor that recognizes conserved motifs on infectious agents; scavenger receptors that recognize diacyl lipids at the bacterium surface; and Toll-like receptors that recognize bacterial structures, such as lipopolysaccharides, peptidoglycan, or flagellin $[47,48]$. The receptor's engagement leads to particle/microorganism internalization through an actin-dependent mechanism [49]. Numerous molecules, such as actinbinding proteins (Arp2/3, dynamin, amphiphysin), membrane traffic regulators (Rho family/GTPase), kinases (PI3K, Hck, etc.), lipases (phopholipase C), and lipids [PI3P, PI $(4,5) \mathrm{P} 2$, etc.] contribute to the internalization of particles/microorganisms. In contrast to macropinocytosis, phagocytosis requires dynamin II, a ubiquitously expressed GTPase that has a critical role in the scission of forming clathrin-coated endocytic vesicles from the plasma membrane and the formation of phagosomes. Indeed, dominant negative forms of dynamin II inhibit phagocytosis at the stage of membrane extension around particles $[3,8,50]$. Recent studies have shown that the endoplasmic reticulum is involved in the phagocytic process $[51,52]$.

After internalization, particles/microorganisms are localized within a phagosome, which then undergoes a series of fusion/fission events with different populations of endosomes. The ultimate stage of these events is the phagolysosome, in which microorganisms are destroyed [53]. The first step of phagosome conversion is the intermingled fusion/fission with early endosomes. Phagosomes gain several markers, such as EEA1 and the small GTPase Rab5. The early phagosomes gradually transform into a compartment with features of late endosomes. Markers of late endosomes, such as the small GTPase Rab7 and lysosomal membrane-associated protein-1 (Lamp-1), progressively replace early endosomal markers $[53,54]$. Whereas the $\mathrm{pH}$ of early phagosomes is around 6.0 , the acquisition of the vacuolar proton pump ATPase $\left(\mathrm{V}-\mathrm{H}^{+}\right.$-ATPase) allows the intraphagosomal $\mathrm{pH}$ to reach 


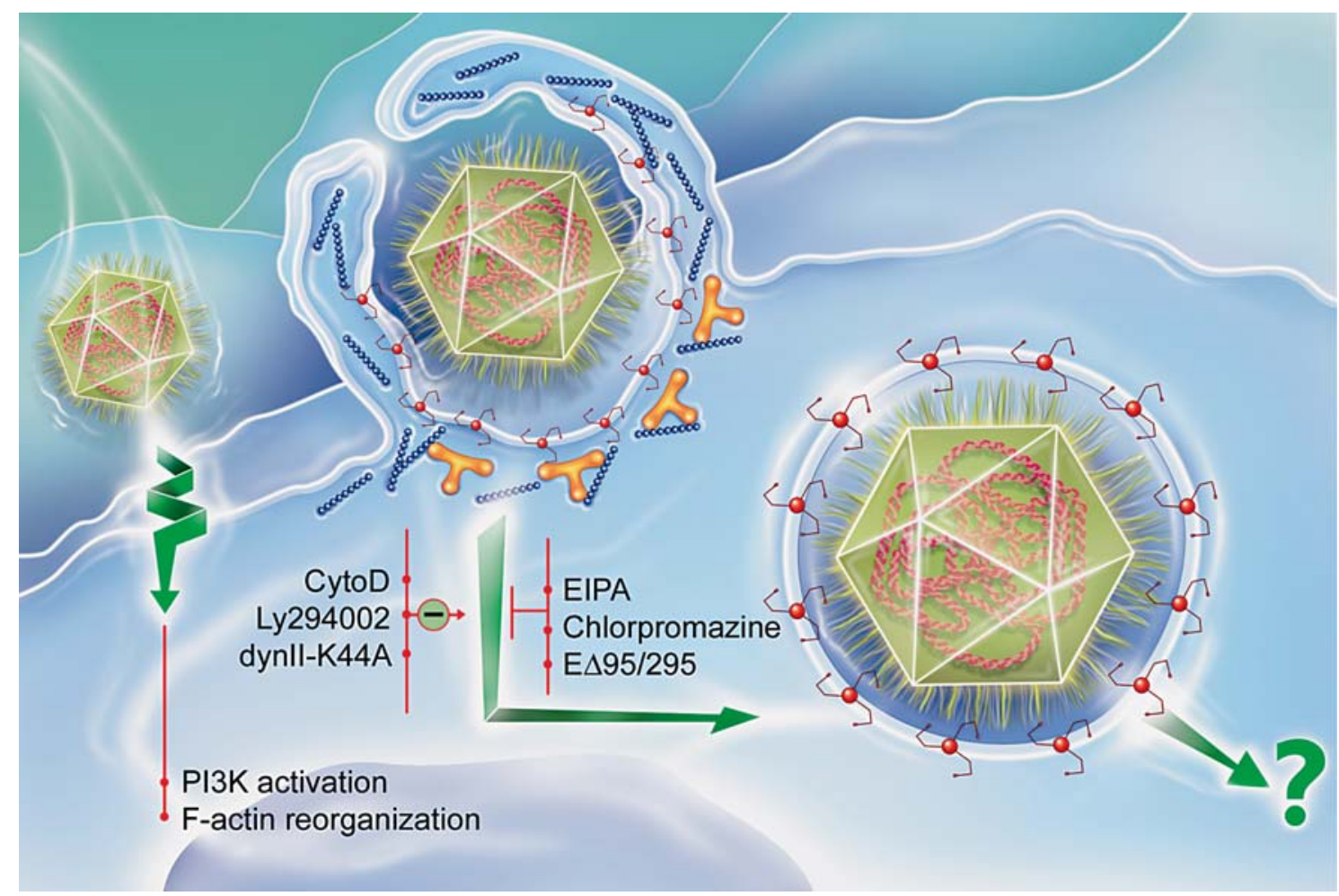

Fig. 2. APMV enters macrophages through a phagocytic process. After binding with macrophages, APMV induces PI3K activation and F-actin polymerization. APMV particles are engulfed by a mechanism involving F-actin (blue), clathrin (red) and dynaminII (yellow). APMV internalization is inhibited by cytochalasin D
(CytoD), Ly294002 and the dominant-negative form of the dynamin-II (dynII-K44A). It is not affected by EIPA, chlorpromazine and dominant-negative form of Eps15 (E $\Delta 95 / 295)$. The mechanism of APMV replication remains unknown. Illustration under Creative Commons Attribution License from [56]. an acidic level of around 4.0 [55]. Finally, late phagosomes interact with lysosomes that contain hydrolytic enzymes such as cathepsin D, thus leading to the formation of phagolysosomes [53, 55].

Phagocytosis is normally used by bacteria, fungi and parasites to infect professional phagocytes. Only recently two studies have demonstrated that phagocytosis is also used by viruses to enter their host cells $[56,57]$ (table 1). Indeed, phagocytosis was suggested for herpes simplex virus (HSV-1) [57] and has been demonstrated for Acanthamoeba polyphaga mimivirus (APMV) [56] as an entry mechanism into their host cells.

HSV-1 infects its target cells, primary human corneal fibroblasts and Chinese hamster ovary cells, through mechanisms that mimic many features of phagocytosis, so called phagocytosis-like, which is not canonical phagocytosis [57] since it takes place in non-professional phagocytic cells. The binding of the HSV-1 envelope to the phagosomal membrane is facilitated by the clustering of nectin-1. During entry, HSV-1 associates with plasma membrane protrusions and is then internalized in a process involving rearrangement of the actin cytoskeleton. Rho GTPases such as RhoA are activated during this process. Moreover, a dynamin II dominant-negative mutant decreases HSV-1 entry. CCV have no detectable role in HSV-1 entry [57].

APMV is a giant double-stranded DNA virus, identified for the first time in 2003 [58], that grows in amoebae. APMV was described to enter professional phagocytes by phagocytosis [56] (fig. 2). Indeed, the engulfment of APMV requires F-actin because cytochalasin D, which inhibits actin polymerization, blocks APMV entry in macrophages in a dose-dependent manner. F-actin labeling with a specific probe and ultrastructural analysis (fig. 3) also showed cell membrane protrusions at the entry site of APMV. APMV does not enter macrophages through macropinocytosis because EIPA, a specific inhibitor of macropinocytosis [31, 32], does not affect 

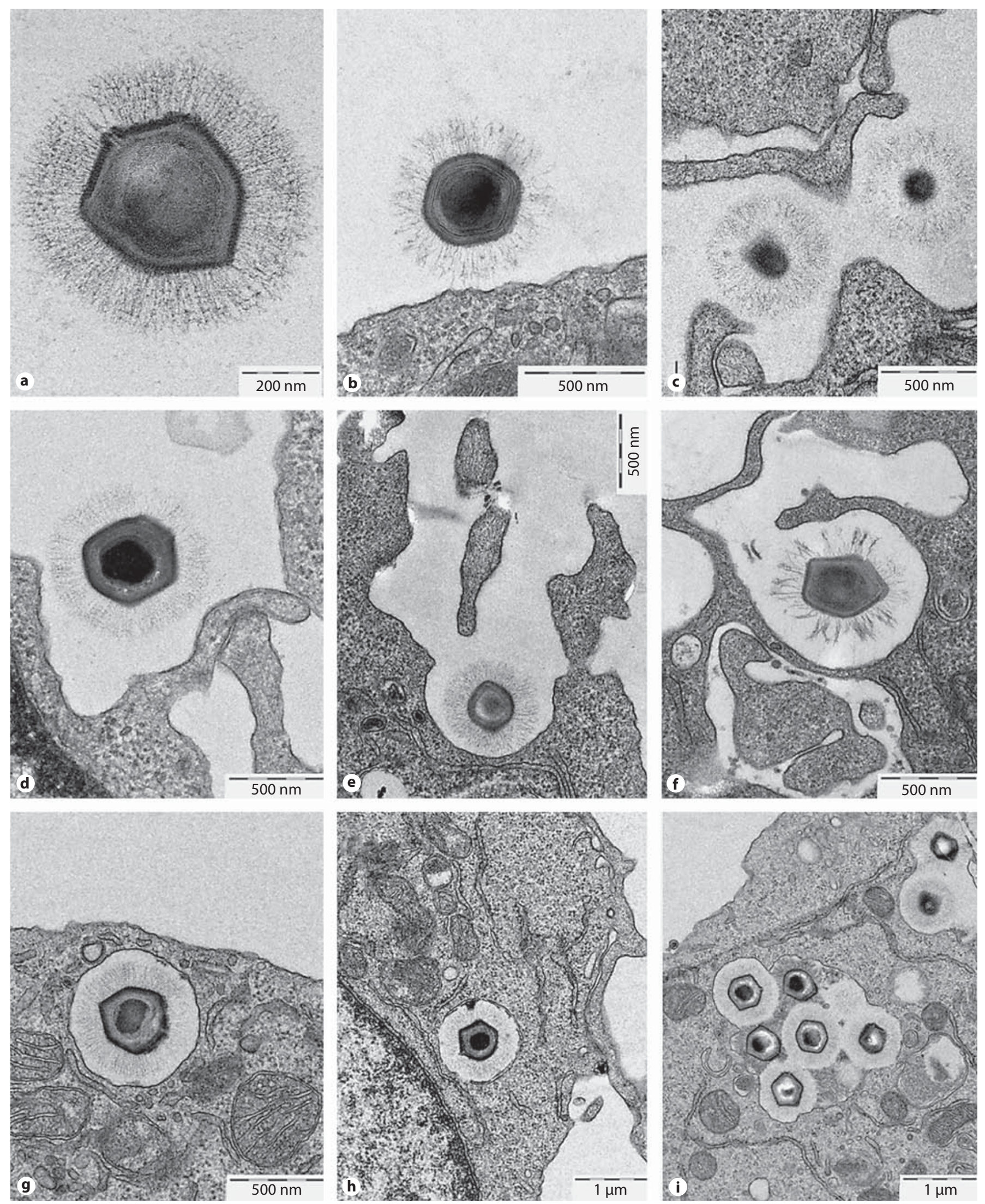
APMV entry into macrophages [56]. Furthermore, rabankyrin-5, a macropinosome marker [33], does not colocalize with APMV. APMV activates PI3Ks, and specific PI3K inhibitors block APMV entry into macrophages. In addition, APMV activates downstream phagocytosis effectors such as Akt and ERK. Moreover, caveolae- and clathrin-dependent endocytic routes, known to be used by viruses to infect cells, are also involved in the entry of APMV [56]. APMV is the first virus described to specifically enter cells by canonical phagocytosis.

\section{Virus Entry in Amoebae}

Amoebae are reservoirs for numerous bacteria [59]; these unicellular organisms are able to hide viruses, particularly giant viruses. For example, amoebae can host giant viruses such as APMV [58] and Mamavirus [60]. The entry process of viruses into amoebae is still unknown. However, it is reasonable to hypothesize that mechanisms of virus internalization in amoebae are similar to those found in mammalian cells. Phagocytosis and macropinocytosis are the principal internalization processes in amoebae. These processes are similar to the mechanisms involved in mammalian cells and are used by amoebae to internalize microorganisms, cells, or apoptotic cells [61-63]. Although phagocytosis is probably the principal internalization mechanism used by amoebae to ingest particles, several studies have highlighted the fact that amoebae are equipped for clathrin- and caveolin-mediated endocytosis. Indeed, amoebae are able to internalize transferrin, ferritin, and holotransferrin mainly through CCV and caveolae-like structures [64]. In addition, several key molecules for endocytosis, such as Rab7 and Rab11, have been identified in amoebae $[65,66]$. It is likely that amoebae are

Fig. 3. Electron microscopic analysis of APMV internalization. The sequence of APMV internalization is illustrated as follows. a Isolated APMV. b APMV bound to the cell body. c APMV attached to cellular extensions. d, e Cup-like indentation formed at the cell surface and large cellular extensions starting to embrace APMV. f Engulfed APMV. g Large smooth-surfaced endocytic vesicle containing APMV. $\mathbf{h}$ APMV-containing vesicle deeper in the cytoplasm. i APMV-containing vesicles that occasionally fused with each other. Illustration under Creative Commons Attribution License from [56].

Virus Entry Pathways equipped to perform endocytosis and phagosome/endosome conversion. It is important to note that the viruses actually discovered in amoebae are giant viruses, suggesting that internalization mechanisms used by amoebae are the base of tropism of giant viruses in these organisms.

\section{Concluding Remarks}

It is now clear that, besides caveolin- and clathrin-mediated endocytosis, viruses use macropinocytosis, phagocytosis or alternative pathways such as late endosomemediated endocytosis (fig. 1). Viruses can use more than one pathway to enter cells, so they are always able to have a host cell to infect. Indeed, influenza viruses, which can use CME (see above) [15], are also able to enter cells in the absence of clathrin [67]. The exact way used by influenza viruses to enter cells in the absence of clathrin is unknown, but it is hypothesized that they use the poorly described non-clathrin- or non-caveolin-dependent endocytosis. HIV, which enters cells by direct fusion with the cell plasma membrane, also appears able to enter cells by macropinocytosis, although this possibility is still under discussion [43-45]. Vesicular stomatitis virus is described as entering cells by CME and infecting cells through a degradative endosome-mediated endocytotic process [68].

Viruses and bacterial pathogens share some entry pathways or molecules involved in their entry. Indeed, HSV-1 and APMV enter cells by phagocytosis, as do numerous bacteria [56, 57]. Bacterial pathogens such as Salmonella, Shigella, Yersinia, and Listeria use CME to enter non-phagocytic cells [69], an entry pathway classically used by viruses [1]. In addition, caveolin was recently shown to be involved in phagocytosis [70]. The fact that viruses use an entry door common to bacteria, like phagocytosis, suggests that viruses have adapted their entry strategies to infect several types of cells. It is important to note that the virulence trait of a virus is not only associated with its multiplication potential but also with the fact that viruses can use numerous entry pathways to enter several cell types. There is a selection of virulence traits in the adaptation of viruses to cells. Certain viruses used several ways to enter cells, with more or less efficiency, highlighting the high infectivity potential of viruses and their adaptive potential. Presumably, other endocytic processes that are unknown or not well characterized are used by viruses. Thus, viruses could be used to identify or characterize endocytic pathways $[8,71]$.

Intervirology 2010;53:274-283 


\section{References}

1 Marsh M, Helenius A: Virus entry: open Sesame. Cell 2006;124:729-740.

$>2$ Takei K, Haucke V: Clathrin-mediated endocytosis: membrane factors pull the trigger. Trends Cell Biol 2001;11:385-391.

$\checkmark 3$ Conner SD, Schmid SL: Regulated portals of entry into the cell. Nature 2003;422:37-44.

$>4$ Doherty GJ, McMahon HT: Mechanisms of endocytosis. Ann Rev Biochem 2009;78: 857-902.

$>5$ Benmerah A, Bayrou M, Cerf-Bensussan N, Dautry-Varsat A: Inhibition of clathrincoated pit assembly by an Eps15 mutant. J Cell Sci 1999;112:1303-1311.

6 Damke H, Baba T, Warnock DE, Schmid SL: Induction of mutant dynamin specifically blocks endocytic coated vesicle formation. J Cell Biol 1994;127:915-934.

7 Sun X, Yau VK, Briggs BJ, Whittaker GR: Role of clathrin-mediated endocytosis during vesicular stomatitis virus entry into host cells. Virology 2005;338:53-60.

$>8$ Pelkmans L, Helenius A: Insider information: what viruses tell us about endocytosis. Curr Opin Cell Biol 2003;15:414-422.

$>9$ Nemerow GR: Cell receptors involved in adenovirus entry. Virology 2000;274:1-4.

10 Smith AE, Helenius A: How viruses enter animal cells. Science (New York, NY) 2004 304:237-242.

11 Rauma T, Tuukkanen J, Bergelson JM, Denning G, Hautala T: Rab5 GTPase regulates adenovirus endocytosis. J Virol 1999;73: 9664-9668.

-12 Kelkar SA, Pfister KK, Crystal RG, Leopold PL: Cytoplasmic dynein mediates adenovirus binding to microtubules. J Virol 2004;78 10122-10132.

$\checkmark 13$ Pekosz A, Newby C, Bose PS, Lutz A: Sialic acid recognition is a key determinant of influenza A virus tropism in murine trachea epithelial cell cultures. Virology 2009;386: 61-67.

14 Sieczkarski SB, Whittaker GR: Differential requirements of Rab5 and Rab7 for endocytosis of influenza and other enveloped viruses. Traffic 2003;4:333-343.

15 Lakadamyali M, Rust MJ, Zhuang X: Endocytosis of influenza viruses. Microbes Infect 2004;6:929-936.

-16 Khor R, McElroy LJ, Whittaker GR: The ubiquitin-vacuolar protein sorting system is selectively required during entry of influenza virus into host cells. Traffic 2003;4:857868.

17 Parton RG, Simons K: The multiple faces of caveolae. Nat Rev 2007;8:185-194.

-18 Ortegren U, Karlsson M, Blazic N, Blomqvist M, Nystrom FH, Gustavsson J, Fredman P, Stralfors P: Lipids and glycosphingolipids in caveolae and surrounding plasma membrane of primary rat adipocytes. Eur J Biochem 2004;271:2028-2036.

19 Pelkmans L, Helenius A: Endocytosis via caveolae. Traffic 2002;3:311-320.
20 Orth JD, McNiven MA: Dynamin at the actin-membrane interface. Curr Opin Cell Biol 2003;15:31-39.

-21 Sharma DK, Brown JC, Choudhury A, Peterson TE, Holicky E, Marks DL, Simari R, Parton RG, Pagano RE: Selective stimulation of caveolar endocytosis by glycosphingolipids and cholesterol. Mol Biol Cell 2004;15:31143122 .

22 Schelhaas M, Malmstrom J, Pelkmans L, Haugstetter J, Ellgaard L, Grunewald K, Helenius A: Simian virus 40 depends on ER protein folding and quality control factors for entry into host cells. Cell 2007;131:516-529.

23 Pelkmans L, Kartenbeck J, Helenius A: Caveolar endocytosis of simian virus 40 reveals a new two-step vesicular-transport pathway to the ER. Nat Cell Biol 2001;3:473-483.

24 Stang E, Kartenbeck J, Parton RG: Major histocompatibility complex class I molecules mediate association of SV40 with caveolae. Mol Biol Cell 1997;8:47-57.

25 Breau WC, Atwood WJ, Norkin LC: Class I major histocompatibility proteins are an essential component of the simian virus 40 receptor. J Virol 1992;66:2037-2045.

26 Richards AA, Stang E, Pepperkok R, Parton RG: Inhibitors of cop-mediated transport and cholera toxin action inhibit simian virus 40 infection. Mol Biol Cell 2002;13:17501764 .

27 Pietiainen V, Marjomaki V, Upla P, Pelkmans L, Helenius A, Hyypia T: Echovirus 1 endocytosis into caveosomes requires lipid rafts, dynamin II, and signaling events. Mol Biol Cell 2004;15:4911-4925.

28 Swanson JA, Watts C: Macropinocytosis. Trends Cell Biol 1995;5:424-428.

29 Racoosin EL, Swanson JA: Macrophage colony-stimulating factor (rM-CSF) stimulates pinocytosis in bone marrow-derived macrophages. J Exp Med 1989;170:1635-1648.

30 Bar-Sagi D, Feramisco JR: Induction of membrane ruffling and fluid-phase pinocytosis in quiescent fibroblasts by Ras proteins. Science 1986;233:1061-1068.

-31 Kee SH, Cho EJ, Song JW, Park KS, Baek LJ, Song KJ: Effects of endocytosis inhibitory drugs on rubella virus entry into VeroE6 cells. Microbiol Immunol 2004;48:823-829.

32 West MA, Bretscher MS, Watts C: Distinct endocytotic pathways in epidermal growth factor-stimulated human carcinoma A431 cells. J Cell Biol 1989;109:2731-2739.

33 Schnatwinkel C, Christoforidis S, Lindsay MR, Uttenweiler-Joseph S, Wilm M, Parton RG, Zerial M: The Rab5 effector rabankyrin-5 regulates and coordinates different endocytic mechanisms. PLoS Biology 2004; 2:E261.
34 Hooshmand-Rad R, Claesson-Welsh L, Wennstrom S, Yokote K, Siegbahn A, Heldin $\mathrm{CH}$ : Involvement of phosphatidylinositide 3'-kinase and Rac in platelet-derived growth factor-induced actin reorganization and chemotaxis. Exp Cell Res 1997;234:434-441.

35 West MA, Prescott AR, Eskelinen EL, Ridley AJ, Watts C: Rac is required for constitutive macropinocytosis by dendritic cells but does not control its downregulation. Curr Biol 2000;10:839-848.

36 Sieczkarski SB, Whittaker GR: Dissecting virus entry via endocytosis. J Gen Virol 2002;83:1535-1545.

37 Li G, D’Souza-Schorey C, Barbieri MA, Cooper JA, Stahl PD: Uncoupling of membrane ruffling and pinocytosis during Ras signal transduction. J Biol Chem 1997;272:1033710340.

-38 Sun P, Yamamoto H, Suetsugu S, Miki H, Takenawa T, Endo T: Small GTPase Rah/ Rab34 is associated with membrane ruffles and macropinosomes and promotes macropinosome formation. J Biol Chem 2003; 278:4063-4071.

39 Mercer J, Helenius A: Vaccinia virus uses macropinocytosis and apoptotic mimicry to enter host cells. Science 2008;320:531-535.

40 Amstutz B, Gastaldelli M, Kalin S, Imelli N, Boucke K, Wandeler E, Mercer J, Hemmi S, Greber UF: Subversion of CtBP1-controlled macropinocytosis by human adenovirus serotype 3. EMBO J 2008;27:956-969.

-41 Wickham TJ, Mathias P, Cheresh DA, Nemerow GR: Integrins alpha v beta 3 and alpha $\mathrm{v}$ beta 5 promote adenovirus internalization but not virus attachment. Cell 1993;73:309319.

42 Sirena D, Lilienfeld B, Eisenhut M, Kalin S, Boucke K, Beerli RR, Vogt L, Ruedl C, Bachmann MF, Greber UF, Hemmi S: The human membrane cofactor CD46 is a receptor for species B adenovirus serotype 3. J Virol 2004; 78:4454-4462.

43 Nguyen DG, Wolff KC, Yin H, Caldwell JS, Kuhen KL: 'UnPAKing' human immunodeficiency virus (HIV) replication: using small interfering RNA screening to identify novel cofactors and elucidate the role of group I PAKS in HIV infection. J Virol 2006;80:130 137.

44 Fontenot DR, den Hollander P, Vela EM, Newman R, Sastry JK, Kumar R: Dynein light chain 1 peptide inhibits human immunodeficiency virus infection in eukaryotic cells. Biochem Biophys Res Commun 2007; 363:901-907.

45 Marechal V, Prevost MC, Petit C, Perret E, Heard JM, Schwartz O: Human immunodeficiency virus type 1 entry into macrophages mediated by macropinocytosis. J Virol 2001; 75:11166-11177.

-46 Aderem A, Underhill DM: Mechanisms of phagocytosis in macrophages. Ann Rev Immunol 1999;17:593-623. 
-47 Taylor PR, Martinez-Pomares L, Stacey M, Lin HH, Brown GD, Gordon S: Macrophage receptors and immune recognition. Ann Rev Immunol 2005;23:901-944.

-48 Stuart LM, Ezekowitz RA: Phagocytosis: elegant complexity. Immunity 2005;22:539550.

49 Underhill DM, Ozinsky A: Phagocytosis of microbes: complexity in action. Ann Rev Immunol 2002;20:825-852.

50 Gold ES, Underhill DM, Morrissette NS, Guo J, McNiven MA, Aderem A: Dynamin 2 is required for phagocytosis in macrophages. J Exp Med 1999;190:1849-1856.

51 Desjardins M: ER-mediated phagocytosis: a new membrane for new functions. Nat Rev Immunol 2003;3:280-291.

-52 Roy CR, Salcedo SP, Gorvel JP: Pathogen-endoplasmic-reticulum interactions: in through the out door. Nat Rev Immunol 2006;6:136-147.

53 Haas A: The phagosome: compartment with a license to kill. Traffic 2007;8:311-330.

-54 Henry RM, Hoppe AD, Joshi N, Swanson JA: The uniformity of phagosome maturation in macrophages. J Cell Biol 2004;164:185-194.

55 Scott CC, Botelho RJ, Grinstein S: Phagosome maturation: a few bugs in the system. J Membr Biol 2003;193:137-152.

56 Ghigo E, Kartenbeck J, Lien P, Pelkmans L, Capo C, Mege JL, Raoult D: Ameobal pathogen mimivirus infects macrophages through phagocytosis. PLoS pathogens 2008; 4:e1000087.

-57 Clement C, Tiwari V, Scanlan PM, Valyi-Nagy T, Yue BY, Shukla D: A novel role for phagocytosis-like uptake in herpes simplex virus entry. J Cell Biol 2006;174:1009-1021.
8 La Scola B, Audic S, Robert C, Jungang L, de Lamballerie X, Drancourt M, Birtles R, Claverie JM, Raoult $\mathrm{D}$ : A giant virus in amoebae. Science 2003;299:2033.

59 Greub G, Raoult D: Microorganisms resistant to free-living amoebae. Clin Microbiol Rev 2004;17:413-433.

60 Raoult D, Forterre P: Redefining viruses: lessons from mimivirus. Nat Rev Microbiol 2008;6:315-319.

61 Labruyere E, Guillen N: Host tissue invasion by Entamoeba histolytica is powered by motility and phagocytosis. Arch Med Res 2006; 37:253-258.

62 Okada M, Nozaki T: New insights into molecular mechanisms of phagocytosis in entamoeba histolytica by proteomic analysis. Arch Med Res 2006;37:244-252.

63 Boettner DR, Huston CD, Linford AS, Buss SN, Houpt E, Sherman NE, Petri WA Jr: Entamoeba histolytica phagocytosis of human erythrocytes involves PATMK, a member of the transmembrane kinase family. PLoS Pathogens 2008;4:e8.

64 Lopez-Soto F, Leon-Sicairos N, Reyes-Lopez M, Serrano-Luna J, Ordaz-Pichardo C, PinaVazquez C, Ortiz-Estrada G, de la Garza M: Use and endocytosis of iron-containing proteins by Entamoeba histolytica trophozoites. Infect Genet Evol 2009;9:1038-1050.

65 McGugan GC Jr, Temesvari LA: Characterization of a Rab11-like GTPase, ehrab11, of Entamoeba histolytica. Mol Biochem Parasitol 2003;129:137-146.
66 Saito-Nakano Y, Mitra BN, Nakada-Tsukui K, Sato D, Nozaki T: Two Rab7 isotypes, EhRab7A and EhRab7B, play distinct roles in biogenesis of lysosomes and phagosomes in the enteric protozoan parasite Entamoeba histolytica. Cell Microbiol 2007;9:17961808.

67 Sieczkarski SB, Whittaker GR: Influenza virus can enter and infect cells in the absence of clathrin-mediated endocytosis. J Virol 2002;76:10455-10464.

68 Le Blanc I, Luyet PP, Pons V, Ferguson C, Emans N, Petiot A, Mayran N, Demaurex N, Faure J, Sadoul R, Parton RG, Gruenberg J: Endosome-to-cytosol transport of viral nucleocapsids. Nat Cell Biol 2005;7:653-664.

69 Veiga E, Cossart P: The role of clathrin-dependent endocytosis in bacterial internalization. Trends Cell Biol 2006;16:499-504.

70 Li J, Scherl A, Medina F, Frank PG, Kitsis RN, Tanowitz HB, Sotgia F, Lisanti MP: Impaired phagocytosis in caveolin-1 deficient macrophages. Cell Cycle 2005;4:1599-1607.

-71 Damm EM, Pelkmans L: Systems biology of virus entry in mammalian cells. Cell Microbiol 2006;8:1219-1227.

72 Empig CJ, Goldsmith MA: Association of the caveola vesicular system with cellular entry by filoviruses. J Virol 2002;76:5266-5270.

73 Richterova Z, Liebl D, Horak M, Palkova Z, Stokrova J, Hozak P, Korb J, Forstova J: Caveolae are involved in the trafficking of mouse polyomavirus virions and artificial VP1 pseudocapsids toward cell nuclei. J Virol 2001;75:10880-10891.

74 DeTulleo L, Kirchhausen T: The clathrin endocytic pathway in viral infection. EMBO J 1998;17:4585-4593. 